\title{
Analysis of Nonlinear Compensation Algorithm for NU150E25TR Ultrasonic Sensor
}

\author{
Longbing Liang ${ }^{1, a}$, Yang Li ${ }^{1, b}$, Lijuang Tang ${ }^{1, c}$, Hui Liü ${ }^{2, d}$ \\ 1 Jiangsu Vocatioal College of business ,nantong,226011,China \\ ${ }^{2}$ China University of Mining and Technology(xuzhou)College,xuzhou,221116,China \\ a807193907@qq.com,b123857099@qq.com,c76462456@qq.com, ${ }^{\mathrm{d}} 120238123 @ q q . c o m$
}

\begin{abstract}
According to the characteristics of the commonly used wind measuring ultrasonic sensor NU150E25TR in the market, the main reasons for the error are analyzed. The characteristics of several main nonlinear compensation algorithms such as linear regression method, least square method and fold line method are compared, and the measured data of single chip microcomputer are used as samples, and the conditions of order and mode are fitted according to different curves. The error of these algorithms is compared and analyzed. The test results show that the piecewise fold fitting method is both simple and effective to reduce the compensation error. Under the same conditions, the accuracy is higher than the linear regression method and the least square method.
\end{abstract}

Keywords: ultrasonic wind speed sensor, wind speed measurement, nonlinearity, compensation algorithm.

\section{Introduction}

There are various kinds of wind speed sensors on the market. There are three kinds of wind speed sensors at home and abroad. The first kind is the propeller wind direction and wind speed sensor; the second type is the wind speed is mode of three cups, the wind direction is the single wing wind direction and wind speed sensor; the third kind is the ultrasonic wind direction wind speed sensor.

The wind speed sensors with obvious advantages and disadvantages or too high price, too high precision and too little precision error.However NU150E25TR sensor can hardly have both precision and price. But in some operating environment with needing higher precision, the precision of NU150E25TR sensor will be insufficient and the cost problem is used in view of the use of the sensor. The algorithm is improved to reduce the cost of design. Combining with the specific software design, this paper chooses linear regression method,the least square method, the fold line method and other main nonlinear compensation algorithms, and designs the hardware circuit.

\section{The Working Principle of the Wind Speed Sensor}

The design uses the ultrasonic vortex transducer to obtain the wind speed. A vortex rod is arranged between the wind sensor transducer (blocking body). when the flow of air cross the vortex rod, it will below the two column rotary alternating vortex. Due to the vortex to the effect of blocking, the ultrasonic transducer will receive ultrasonic intensity changing with the vortex frequency of vortex.when no ultrasonic barrier, the received ultrasonic intensity is maximum; when the vortex is blocking the ultrasonic, the received ultrasonic strength. The ultrasonic transducer converts the received frequency signal to the frequency / voltage conversion module and converts it into voltage, which is linearly proportional to the two theoretically, that is, voltage and wind speed are linearly proportional. Its schematic diagram is shown in Figure 1. 


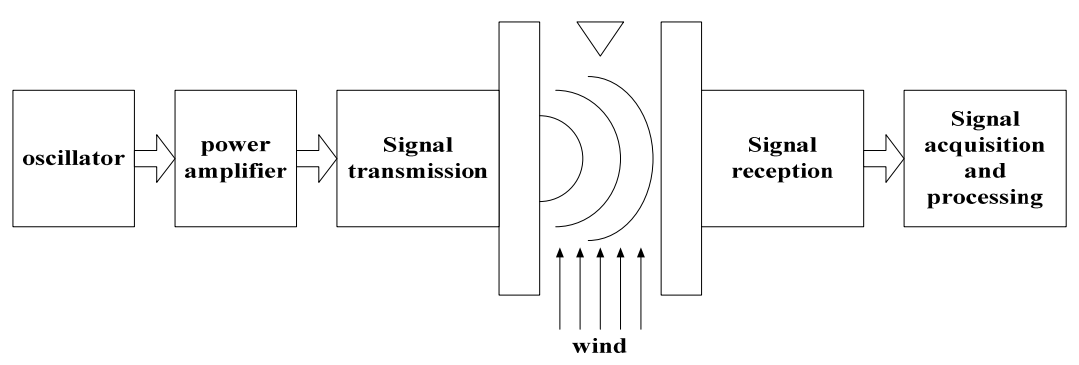

Fig 1.principle of ultrasonic transmitting and receiving

This design uses STC90C58AD chip as the main control module. The frequency of STC90C58AD chip range 0-40MHz, with EEpROM, watchdog function, 316 bit timing / counters, 8 - way 10 - bit AD sampling and conversions and so on.

From Fig 2, we can know that the whole system mainly consists of ultrasonic signal transmitter / receiver module, frequency / voltage conversion module, output module, watchdog module, infrared remote control module and LCD module. Through the signal transmitting module to the ultrasonic transducer 1 to provide incentives to transmit ultrasonic signal, ultrasonic transducer 2 will receive ultrasonic signal intensity changes with the vortex frequency and signal into frequency / voltage conversion module is converted into a corresponding voltage, and came into the MCU to start $\mathrm{AD}$ sampling. Finally through the MCU processing, the output of wind speed, air volume, to achieve a variety of output such as the frequency, current output was came true, and also can realize the infrared remote control and remote transmission function diversity. The data used in this article are obtained through this device.

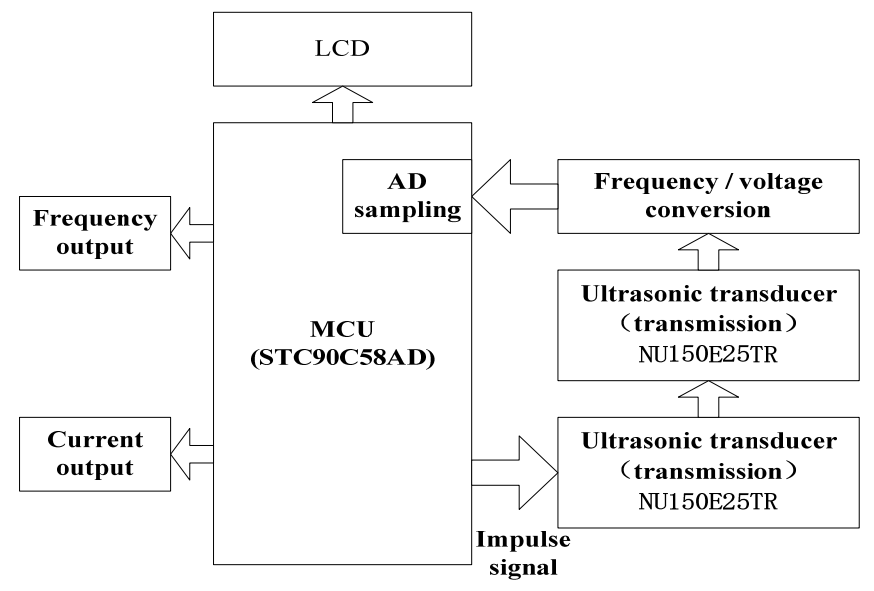

Fig 2.wind measurement system

\subsection{The Characteristics of NU150E25TR Supersonic Wave Sensor}

As shown in Fig 3, the following data can be determined by the test model of the NU150E25TR ultrasonic sensor. The NU150E25TR ultrasonic sensor is a high frequency type, the signal is transfered and received in one. its nominal frequency is $150 \pm 8.0 \mathrm{KHz}$, and the bandwidth is $12.0 \mathrm{KHz}$. Its sensitivity is $-72 \mathrm{~dB}$ min , maximum input voltage $500 \mathrm{Vp}-\mathrm{p}$, direction angle is $14 \pm 2$ degrees, detection range: $0.1 \sim 2 \mathrm{~m}$ (reflection), working temperature is $-20^{\circ} \mathrm{C} \sim+80^{\circ} \mathrm{C}$, storage temperature is -40 degree ${ }^{\circ} \mathrm{C} \sim+85^{\circ} \mathrm{C}$, and is the market commonly used wind sensor. 


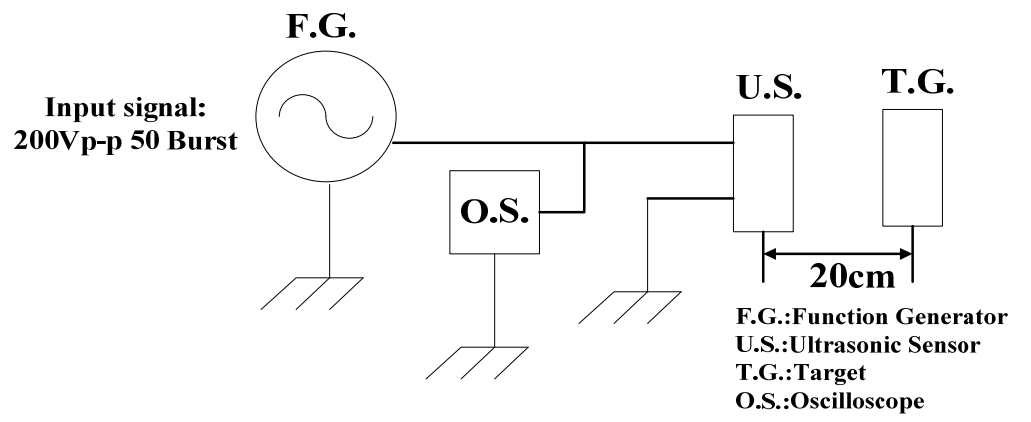

Fig 3. NU150E25TR-1 test model

The test relies on the main environmental is wind tunnel, and other standard wind measuring instruments. The test wind tunnel is located in the space closed workshop. The outside disturbance is small. The range of wind speed that the wind transport equipment can provide is $0-18 \mathrm{~m} / \mathrm{s}$. The wind measuring reference instrument is Taiwan Tai Shi wind meter instrument. The field wind speed is $0 \mathrm{~m} / \mathrm{s}$, which ensures the accuracy of the data obtained in the wind measurement environment when the air transmission equipment is not started.

From the foregoing, we can know that the principle of this design is a linear relationship between wind speed and ultrasonic frequency,and transform the ultrasonic frequency into the corresponding $A D$ value, and further get the wind speed through the linear relationship between $A D$ value and wind speed. But in the measured data, the relationship between the two is not absolutely linear.So in the measurement time, we was only in the liquid crystal display interface on the windfinding system display $\mathrm{AD}$ value of wind speed and records at the same time Taishi wind instrument display, taking into account the amount of computation based on single chip, using the compensation algorithm. This paper has done similar project, obtained the measured data.

\subsection{Nonlinear Compensation Algorithm}

In the ideal state, the relationship between the wind speed and the $\mathrm{AD}$ value is linear, and the specific relationship is shown as (1).

$$
v=\sum_{i=1}^{n} k a(i)
$$

Where $\mathrm{k}$ is a constant, in this formula, a (i) is used to represent the AD value, and the $\mathrm{v}$ is the wind speed. But in the actual process, different devices and environment affect the linear relationship between wind speed and AD value. In order to maintain the accuracy of measurement, the following methods are applied to analyze and research.

\subsubsection{Linear Regression}

To obtain the relationship between wind speed and $\mathrm{AD}$ by linear regression, is a feasible method.The principle is linear regression is called a equation of linear regression using least square function of one or more independent variables and the dependent relationship between variables, modeling a regression analysis. This function is a linear combination of one or more model parameters called regression coefficients. The relationship between the wind speed value and the AD value is established as a linear regression relationship between the two.

The linear equation is analyzed.

$$
h_{\theta}(x)=\theta_{0}+\theta_{1} x
$$

The added variables are as follows:

$$
h(x)=\sum_{i=0}^{n} \theta_{i} x_{i}=\theta^{T} X
$$

The upper form is $\mathrm{x}=1$, and the linear equation is turned into the parameter $\theta^{\mathrm{T}}$ of the equation.Linear regression hypothesis features and results satisfy the linear relationship. In fact, the expression of linear relations is very powerful. The impact of each feature on the results can be 
reflected by the preceding parameters, and each characteristic variable can be first mapped to a function and then involved in linear computing, which can express the non linear relationship between the characteristics and the results. The linear relationship between the AD values and the wind speed is obtained according to the collected data, and the relationship between the two is obtained.

\subsubsection{Least Square Method}

Using the least squares method to reduce the error between the AD value and wind speed, is also a commonly used nonlinear compensation algorithm, in order to determine the equation of a $0, \mathrm{a} 1, \mathrm{yj}$ will be measured (yj=a0+a1xj) type (2) of the deviation (yi-yj) of the square and minimum optimization criterion ", in which yj and $x \mathrm{j}$ were $\mathrm{AD}$ value and the value of wind speed.

$$
\varphi=\sum(Y i-Y j)^{2}
$$

Replace (type 1) into (type 2)

$$
\varphi=\sum\left(Y i-a_{0}-a_{1} X i\right)^{2}
$$

When $\sum(Y i-Y j)^{2}$ is the hourly, the partial derivative can be obtained by the function pair and the two partial derivatives are equal to zero.

$$
\begin{gathered}
\sum 2\left(a_{0}+a_{1} X i\right)^{2}=0(4) \\
\sum 2 X i\left(a_{0}+a_{1} * X i-Y i\right)=0(5)
\end{gathered}
$$

Or

$$
\begin{gathered}
n a_{0}+\left(\sum X i\right) a_{1}=\sum Y i(6) \\
\left(\sum X i\right) a_{0}+\left(\sum X i^{\wedge} 2\right) a_{1}=\sum(X i * Y i)(7)
\end{gathered}
$$

To bring and bring into (2), the 3 equation of regression.

In the regression equation, correlation regression is not possible through each review, data points, in order to determine the correlation quality by correlation coefficient "R", "F" statistics, the residual standard deviation "S" judgment, "R" is close to 1 ; "F" better "S;" the more than 0.

$$
R=\left[\sum X_{i} Y_{i}-m\left(X_{i} / m\right)\left(\sum Y_{i} / m\right)\right] / S Q R\left\{\left[X_{i 2}-m\left(\sum X_{/} / m\right)^{2}\right]\left[\sum Y_{i 2}-m\left(\sum Y_{i} / m\right)^{2}\right]\right\} \operatorname{In}(1-9), \mathrm{m}
$$
is the sample size, that is, the number of tests, and the values of any set experimental data $X$ and $Y$.

\subsection{Fold Line Fitting Algorithm}

Using of polygonal fitting method to reduce the error between AD and wind speed is a commonly used nonlinear compensation algorithm. It uses the 24 spline function and the piecewise interpolation fast calculation method, and uses the fitting method of broken line resampling to remove redundant interpolation points. The algorithm described in this paper uses a folded line resampling to ensure the accuracy of the drawing with the minimum amount of data.

\subsubsection{Piecewise Fitting Method}

piecewise fitting principle is to the point of a series of fixed point $\mathrm{p} 0, \mathrm{p} 1, \mathrm{p} 2, \mathrm{pn}-3, \mathrm{pn}-2, \mathrm{pN}-1, \ldots$, followed by sampling fitting method:

1) fit $\mathrm{p} 0, \mathrm{p} 1, \mathrm{p} 2, \mathrm{p} 3$ to get the points between $\mathrm{p} 1$ and $\mathrm{p} 2: \mathrm{q} 11, \mathrm{q} 12, \mathrm{q} 13, \ldots, \mathrm{q} 1 \mathrm{~m}$.

2) fitting q1m, p2, p3, p4, p2, p3 between the q22, q23, q21, q2n,...

3) fitting q2n, p3, p4, p5, p3, p4 between the q32, q33, q31, q3w,..

4)...

According to the above method, it has been fitted out that the fitting points and the original control points above the points between pn-3 and pn-2 constitute the fitting polygon of the whole function curve in sequence. Sampling this fitting method, the calculation is small, it is very easy to compile the program.

\subsubsection{The Accuracy of Fitting}

However, the accuracy of this fitting is limited above. On the one hand, we want to improve the precision precision, only the step set is very small.We don't want to add a lot of data. 
Usually we only want to meet our requirements at a small number of points in the gentle curve of the curve. And at the turn, fit with a dense point. Therefore, the method of resampling is introduced to remove some unnecessary points. The basis of resampling is:

Set off line on a series of points $\mathrm{p} 0, \mathrm{p} 1, \mathrm{p} 2, \mathrm{pm}, \mathrm{pn}, \ldots$ Calculate the distance of $\mathrm{p} 1, \mathrm{p} 2, \ldots, \mathrm{pm}$ to line $\mathrm{p} 0$, pn,respectively. If the maximum value pk of these distances is less than a certain limit dist, then the point $\mathrm{p} 1, \mathrm{p} 2, \ldots, \mathrm{pm}$ on the curve are not necessary, so we just keep p0, pn.

If $\mathrm{pk}$ is greater than our prescribed limit dist value, then we should take (p0, p1, p2, pk-2, pk-1, $\mathrm{pk})$ and ( $\mathrm{pk}, \mathrm{pk}+1, \mathrm{pk}+2, \ldots, \mathrm{pk}+1)$ as 2 new polylines to recalculate the above.

Here, we are almost perfect to solve the problem: sampling piecewise spline interpolation, and using resampling, reducing the amount of data, the same to ensure the precision loss of.

\subsection{Combination of Least Squares and Fold Line Method}

The best function match is found by minimizing the square of the error and searching for the data. By using the least squares method, the unknown data can be easily obtained, and the sum of the square sum of the error between the obtained data and the actual data is minimized. The combination of the least square method and the fold line method can greatly improve the local or the overall error and accuracy.

\section{Comparison and Analysis of Data Error of Nonlinear Compensation Algorithm}

The test results in this paper, the test environment is composed of a powerful fan and wind tunnel barrel tube, test equipment using hardware 51 chip to NU150E25TR data acquisition measurement of ultrasonic sensor, the wind meter data collection promulgated by the state standard for comparison, data is in contrast to the $\mathrm{AD}$ value and real wind speed. In fact, the measured data takes the average of ten values.

\subsection{Data and Error by Using Linear Regression}

Table 1.The relationship between the theoretical value and the actual measured value

\begin{tabular}{cccccccc}
\hline \multirow{2}{*}{ wind speed(m/s) } & $\begin{array}{c}\text { Theoretical } \\
\text { AD value }\end{array}$ & $\begin{array}{c}\text { MD value } \\
\text { AD }\end{array}$ & Eror $(\%)$ & wind speed(m/s) & \multicolumn{2}{c}{ Theoretical } & Measured \\
AD value & AD value & Error(\%) \\
\hline 0 & 47.35 & 15 & 68.32 & 9 & 475.37 & 503 & 68.32 \\
0.5 & 71.13 & 42 & 40.95 & 10 & 522.93 & 520 & 40.95 \\
1 & 94.91 & 74 & 22.03 & 10.5 & 546.71 & 542 & 22.03 \\
1.5 & 118.69 & 107 & 9.85 & 11 & 570.49 & 565 & 9.85 \\
2 & 142.47 & 143 & -0.37 & 11.5 & 594.27 & 595 & -0.37 \\
3 & 190.02 & 202 & -6.30 & 12 & 618.05 & 630 & -6.30 \\
4 & 237.58 & 252 & -6.07 & 13 & 665.60 & 667 & -6.07 \\
5 & 285.14 & 309 & -8.37 & 14 & 713.16 & 712 & -8.37 \\
6 & 332.70 & 357 & -7.30 & 15 & 760.72 & 755 & -7.30 \\
7 & 380.26 & 405 & -6.51 & 16 & 808.28 & 778 & -6.51 \\
8 & 423.26 & 451 & -18.60 & 17.6 & 884.37 & 864 & -18.60 \\
\hline
\end{tabular}




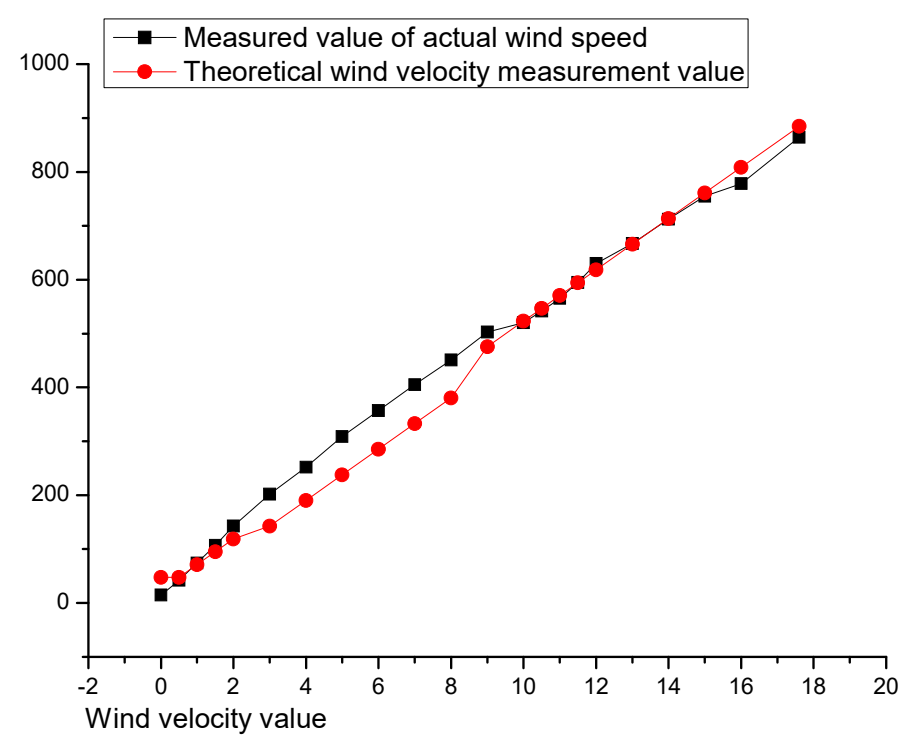

Fig 4.Comparison of the relationship between theoretical values and actual measured values

From Table 1 and Fig 4,The average error of the linear regression is about 4.04\%, and the standard deviation is 23.189. As shown in Figure 4, there is a larger deviation when the wind speed is $2 \mathrm{~m} / \mathrm{s}$ to $10 \mathrm{~m} / \mathrm{s}$.

\subsection{Data and Error Measured by Least Square Method}

Table 2.The relationship between the theoretical value and the actual measured value

\begin{tabular}{cccccccc}
\hline \multirow{2}{*}{ wind speed $(\mathrm{m} / \mathrm{s})$} & $\begin{array}{c}\text { Theoretical } \\
\text { AD value }\end{array}$ & $\begin{array}{c}\text { Measured } \\
\text { AD value }\end{array}$ & error $(\%)$ & wind speed $(\mathrm{m} / \mathrm{s})$ & \multicolumn{2}{c}{$\begin{array}{c}\text { Theoretical } \\
\text { AD value }\end{array}$} & $\begin{array}{c}\text { Measured } \\
\text { AD value }\end{array}$ \\
\hline 0 & 47.35 & 15 & 82.81 & 9 & 475.39 & 503 & -480.15 \\
0.5 & 71.13 & 42 & 51.86 & 10 & 522.95 & 520 & -500.19 \\
1 & 94.91 & 74 & 15.15 & 10.5 & 546.73 & 542 & -525.81 \\
1.5 & 118.69 & 107 & -22.74 & 11 & 570.51 & 565 & -552.61 \\
2 & 142.47 & 143 & -64.10 & 11.5 & 594.29 & 595 & -587.51 \\
3 & 190.03 & 202 & -131.97 & 12 & 618.07 & 630 & -628.22 \\
4 & 237.59 & 252 & -189.59 & 13 & 665.63 & 667 & -671.55 \\
5 & 285.15 & 309 & -255.36 & 14 & 713.19 & 712 & -724.21 \\
6 & 332.71 & 357 & -310.86 & 15 & 760.75 & 755 & -774.62 \\
7 & 380.27 & 405 & -366.44 & 16 & 808.31 & 778 & -801.93 \\
8 & 427.83 & 451 & -419.79 & 17.6 & 884.41 & 864 & -902.80 \\
\hline
\end{tabular}




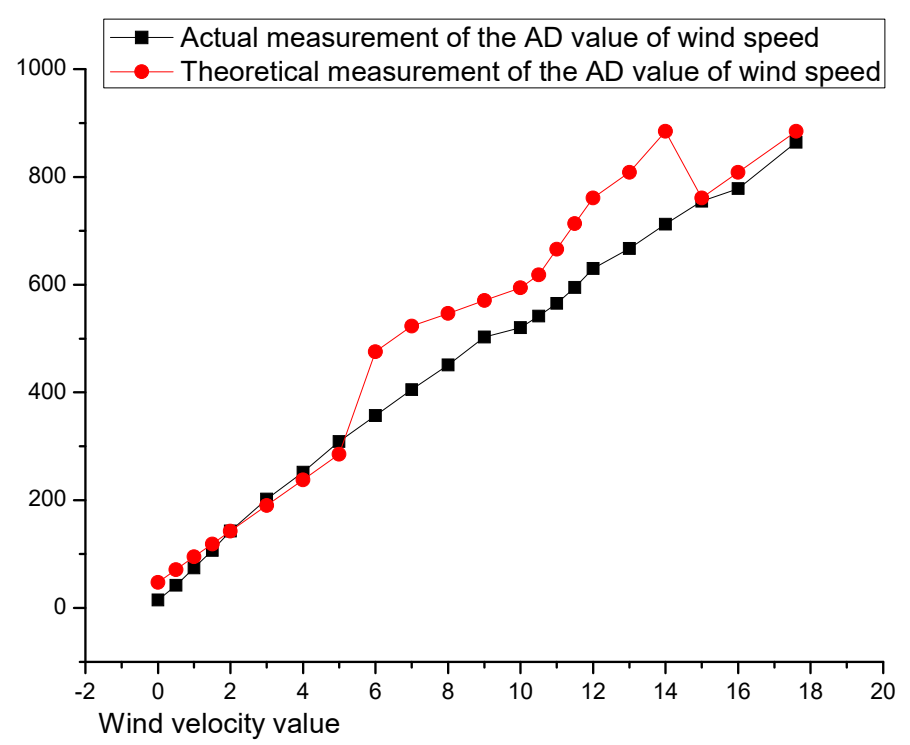

Fig 5. Comparison of the relationship between theoretical values and actual measured values

From Table 2 and Fig 5, The average error of the linear regression is about 4.64\%, and the standard deviation is 342.772 . If the wind speed is $6 \mathrm{~m} / \mathrm{s}$ to $16 \mathrm{~m} / \mathrm{s}$, there is a certain error, as shown in Figure 5 .

\subsection{The Data and Error Measured by the Fitting Algorithm of the Fold Line}

Table 3.The relationship between the theoretical value and the actual measured value

\begin{tabular}{cccccccc}
\hline wind speed $(\mathrm{m} / \mathrm{s})$ & $\begin{array}{c}\text { Theoretical } \\
\text { AD value }\end{array}$ & $\begin{array}{c}\text { Measured } \\
\text { AD value }\end{array}$ & error & wind speed $(\mathrm{m} / \mathrm{s})$ & $\begin{array}{c}\text { Theoretical } \\
\text { AD value }\end{array}$ & $\begin{array}{c}\text { Measured } \\
\text { AD value }\end{array}$ & error \\
\hline 0 & 14.5 & 15 & -0.5 & 9 & 510.8 & 503 & 7.8 \\
0.5 & 41.5 & 42 & -0.5 & 10 & 518.2 & 520 & -1.8 \\
1 & 72.3 & 74 & -1.7 & 10.5 & 540.8 & 542 & -1.2 \\
1.5 & 106.7 & 107 & -0.3 & 11 & 569.6 & 565 & 4.6 \\
2 & 145.5 & 143 & 2.5 & 11.5 & 587.5 & 595 & -7.5 \\
3 & 200 & 202 & -2 & 12 & 625.4 & 630 & -4.6 \\
4 & 254.4 & 252 & 2.4 & 13 & 668.9 & 667 & 1.9 \\
5 & 306.6 & 309 & -2.4 & 14 & 712.7 & 712 & 0.7 \\
6 & 357.2 & 357 & 0.2 & 15 & 753.1 & 755 & -1.9 \\
7 & 402.9 & 405 & -2.1 & 16 & 779.9 & 778 & 1.9 \\
8 & 445.1 & 451 & -5.9 & 17.6 & 865.6 & 864 & 1.6 \\
\hline
\end{tabular}




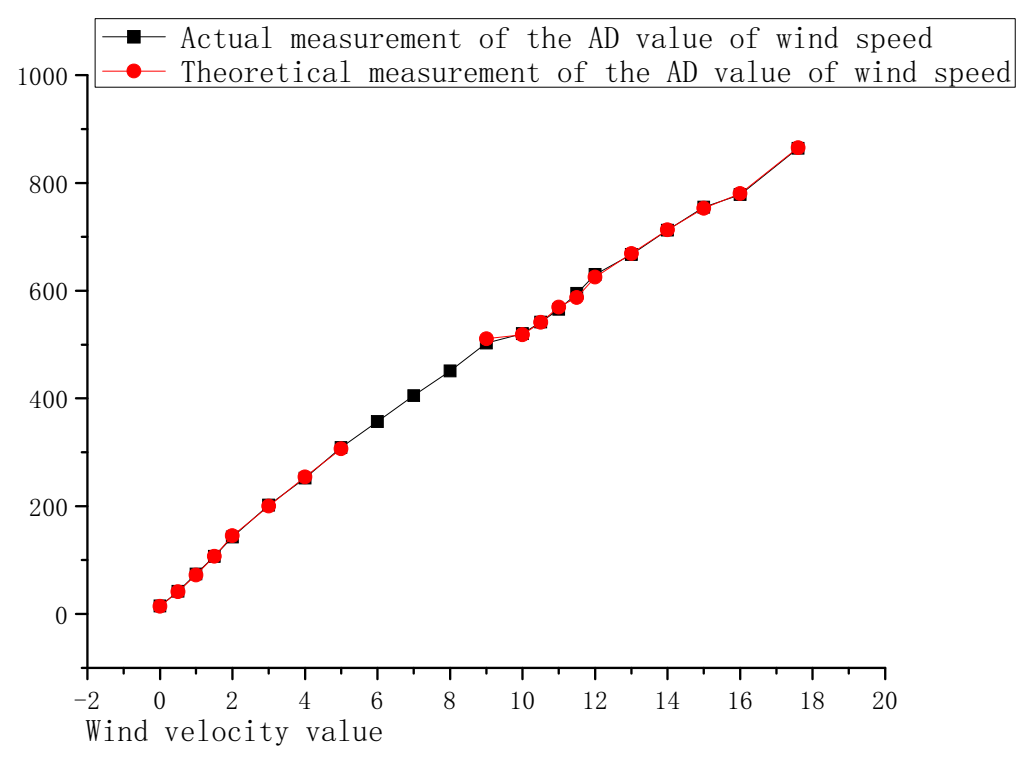

Fig 6. Comparison of the relationship between theoretical values and actual measured values

From Table 3 and Fig 6, The average error of linear regression is about $-0.36 \% \%$, and the standard deviation is 134.875. As shown in Figure 6, the wind speed has no larger error in the whole range.

According to the measured contrast, combined with the average error, the standard deviation and the fitting contrast figures, the method of line fitting is the best method to measure the wind speed, and the error is the least. The method is more suitable than the least square method and linear regression method.

\section{Summary}

By comparing various algorithms, this paper analyzes the main causes of errors, compares the characteristics of several main nonlinear compensation algorithms, such as linear regression, least squares and fold lines, and uses the measured data of single chip as samples, according to the order of order and the way of fitting different curves, the error of these algorithms is compared. Analysis. The test results show that the piecewise least square curve fitting method is simple and effective to reduce the compensation error. Under the same conditions, the accuracy is higher than the Newton iterative method and the look-up table method.

\section{Acknowledgments}

Jiangsu College "Qinglan" Project No.17062006.Nantong Science and Technology Project No.GY12015037.

\section{References}

[1]. Liang L B, Liu H, Zhuo R, et al. Design of Mine Ultrasonic Wind Speed Sensor[J]. Coal Mine Machinery, 2015.

[2]. Zhang D M, Cao X Z, Shang-Chang M A. Design of two-dimensional ultrasonic wave wind speed and wind direction sensor[J]. Transducer \& Microsystem Technologies, 2015.

[3]. Yonehara Y, Goto Y, Yoda K, et al. Flight paths of seabirds soaring over the ocean surface enable measurement of fine-scale wind speed and direction.[J]. Proc Natl Acad Sci U S A, 2016, 113(32):9039-9044. 
[4]. Azorin-Molina C, Vicente-Serrano S M, Mcvicar T R, et al. Assessing the impact of measurement time interval when calculating wind speed means and trends under the stilling phenomenon[J]. International Journal of Climatology, 2017, 37(1).

[5]. Binwei J I, Chen X. Research on Reactive Power Compensation of Distribution Network Based on Particle Swarm Optimization Algorithm[J]. Power System \& Clean Energy, 2016.

[6]. Abdelkareem A E, Sharif B S, Tsimenidis C C. Adaptive time varying doppler shift compensation algorithm for OFDM-based underwater acoustic communication systems[M]. Elsevier Science Publishers B. V. 2016.

[7]. Xie K, Wang K. Measurement of Wind Speed and Direction with Ultrasonic Sensor Using FPGA[J]. Energy Procedia, 2011, 12:837-843. 